THIS IS A PRE-PRINT OF A MANUSCRIPT THAT HAS NOT YET BEEN PEERREVIEWED

\title{
Investigating the Feasibility of Idiographic Network Models
}

\author{
Alessandra C. Mansueto ${ }^{1,2,3}$, Reinout W. Wiers ${ }^{1,3}$, Julia C.M. van Weert ${ }^{1,2}$, Barbara C. \\ Schouten $^{1,2}$, Sacha Epskamp ${ }^{1,3}$ \\ ${ }^{1}$ Centre for Urban Mental Health, Universiteit van Amsterdam \\ ${ }^{2}$ Department of Communication Science, Universiteit van Amsterdam \\ ${ }^{3}$ Department of Psychology, Universiteit van Amsterdam
}

\section{Author Note}

The R code used in this study is shared in the Supplementary Materials. One of the two datasets used in the current study is available in the Supplementary Materials of Fried at al. (2020). This study is based on two datasets used in previous publications (Fried at al., 2020; Piasecki et al., 2011). There is no conflict of interest to disclose. We would like to thank Robert D. Dvorak, Ingmar H. A. Franken, Eiko I. Fried, Chad J. Gwaltney, Thomas M. Piasecki, Kenneth J. Sher, and Andrew J. Waters for sharing with us their data. Additionally, we would like to thank Fabio A. Melis for raising discussion points that were of inspiration for this work during his master thesis. Part of the current work was presented during the Psychological Networks Amsterdam Summer School 2020, and will be presented in a poster at the $54^{\text {th }}$ Annual Convention of the Association for Behavioral and Cognitive Therapies. 


\begin{abstract}
Recent times have seen a call for personalized psychotherapy and tailored communication during treatment, leading to the necessity to model the complex dynamics of mental disorders in a single subject. To this aim, time-series data in one patient can be collected through ecological momentary assessment and analyzed with the graphical vector autoregressive model, estimating temporal and contemporaneous idiographic networks. Idiographic networks graph interindividual processes that may be potentially used to tailor psychotherapy and provide personalized feedback to clients, and are regarded as a promising tool for clinical practice. However, the question whether we can reliably estimate them in clinical settings remains unanswered. We conducted a large-scale simulation study in the context of psychopathology, testing the performance of personalized networks with different numbers of time points, percentages of missing data, and estimation methods. Results indicate that sensitivity is low with sample sizes feasible for clinical practice (75 and 100 time points). It seems possible to retrieve the global network structure, but not to recover the full network. Estimating temporal networks appears particularly challenging, thus with 75 and 100 observations, it is advisable to reduce the number of nodes to around 6 variables. With regard to missing data, FIML and the Kalman filter are effective in addressing random item-level missing data, consequently planned missingness is a valid method to deal with missing data. We discuss possible methodological and clinical solutions to the challenges raised in this work.
\end{abstract}

Keywords: Graphical Vector Autoregressive Model, Ecological Momentary Assessment, Idiographic Network Analysis, Personalized Psychotherapy 


\section{Investigating the Feasibility of Idiographic Network Models}

Two trends are emerging in the field of clinical psychology. First, the call for personalized psychotherapy (Wright \& Woods, 2020), which aims to tailor psychotherapy to the specific needs of a single patient, and can be achieved by incorporating the measurement of intra-individual processes into treatment (Piccirillo \& Rodebaugh, 2019). Second, the necessity to model the complex interactions between psychological symptoms and related factors to predict the development of psychopathology over time, and the effects of treatment on this development, which led to the network theory of psychopathology (Borsboom, 2017; Borsboom \& Cramer, 2013; Cramer et al., 2010; Cramer \& Borsboom, 2015). Idiographic networks have been introduced in psychopathology to model these complex interactions in a single subject and, based on these, adapt psychotherapy (Epskamp, van Borkulo, et al., 2018). Although these developments are exciting, the question remains to what extent it is actually feasible to estimate reliable network structures in a clinical setting. Importantly: previous work on validating these network models rely on synthetic network structures under which network estimation can be expected to work well, and did not investigate expected limitations in clinical data such as missing observations and a low number of responses. We therefore conducted a large-scale simulation study in the context of psychopathology, testing the performance of plausible idiographic network structures with different sample sizes, percentages of missing data, and estimation methods. Our results will lead to recommendations for the application of personalized symptoms networks in clinical research and practice.

\section{The Importance of Idiographic Dynamic Networks}

The network theory of psychopathology assumes that mental disorders emerge from the dynamic interaction between psychological symptoms and the factors influencing these symptoms, and that mental disorders can therefore best be represented as dynamic networks 
(Borsboom, 2017; Borsboom \& Cramer, 2013; Cramer et al., 2010; Cramer \& Borsboom, 2015). Arising from this theoretical view, network analysis has been increasingly used to understand the complex mechanisms of mental disorders (Hofmann et al., 2016; McNally, 2016; for recent reviews of the literature see Fried et al., 2017 and Robinaugh et al., 2020), as it allows to model the multivariate relationships between symptoms as well as protective and risk factors, both internal and external to the individual (e.g., emotions, triggers in the environment). Idiographic networks can potentially be used to model these interactions within one person (Epskamp, van Borkulo, et al., 2018). Since their introduction they have been proposed as a promising method to investigate treatment mechanisms (Hofmann et al., 2020), and a number of studies suggesting their potential to aid personalized psychotherapy has already been published (e.g., Frumkin et al., 2019; Kroeze et al., 2017; Robinaugh, Brown, et al., 2020).

Even though between-subject effects usually cannot be generalized to intra-individual processes (Fisher et al., 2018; Molenaar, 2004), group-level research is predominant in psychology. Studying intra-individual dynamics is also essential, as clinicians need to understand the intra-individual processes involved in the mental health problems of a specific patient (Howard et al., 1996). Recent technological advancements (e.g., smartphones, wearables) enable easy collection of intensive time-series data (Mohr et al., 2017; Shiffman et al., 2008; Trull \& Ebner-Priemer, 2013), thus studying intra-individual dynamics is becoming more common (Hamaker \& Wichers, 2017; Patrick et al., 2016) and incorporating the measurement of intra-individual processes into treatment has been proposed as a promising way forward to improve psychotherapy (Fisher et al., 2019; Iorfino et al., 2019; Piccirillo \& Rodebaugh, 2019; Scott \& Lewis, 2015). This idea is in line with the strong trend towards personalized interventions that is emerging in the fields of clinical and health psychology (Chevance et al., 2020; Wright \& Woods, 2020). 
Commonly used treatments, such as cognitive behavioral therapy, are not equally effective for every client. One of the possible reasons could be that patients sharing the same diagnosis can differ in their psychopathological mechanisms (Fisher \& Bosley, 2015; Fisher \& Boswell, 2016), consequently, their response to treatment may vary. Intensive time-series data can be used to estimate idiographic networks representing the relationship between psychological symptoms and other factors such as social support, physiological activation, and location. These interactions are indicative of individual psychopathological mechanisms and can be discussed with the clients to tailor psychotherapy. For example, if the network shows that for one individual smoking marijuana is followed by an increase in psychosisrelated complaints, which is subsequently followed by an increase in feelings of being discriminated against, which may be followed by an increase in anxiety, which in turns relates to an increase in smoking, then this nodal sequence could be addressed during treatment. The therapist could show the patient that smoking marijuana does not improve anxiety, but rather may even cause it instead, and help the client to develop alternative coping strategies to deal with anxiety and to stop smoking. Idiographic networks are also an intuitive visualization tool, as they clearly graph the negative and positive relationships between variables. They could be used to provide personalized feedback to patients, which is expected to increase motivation to engage with therapy and, consequently, improve outcomes (Birnbaum et al., 2015; Eisen et al., 2000; Hawkins et al., 2008). Therapists may employ networks as a communication apparatus to share knowledge with the clients, actively involve them in the therapeutic process, and receive their feedback. For example, idiographic networks could be estimated before treatment and discussed with the clients to codevelop personalized therapy. During and after therapy, these networks may be estimated again to assess and discuss treatment course and outcome (Von Klipstein, Riese, Servaas \& Schoevers, 2020). 


\section{Modeling Idiographic Dynamic Networks}

Ecological Momentary Assessment (EMA; Shiffman et al., 2008), where individuals answer self-report items multiple times a day for multiple days, usually on their smartphones, is used to collect intensive time-series data. These data can be analyzed to estimate two idiographic dynamic networks: the temporal and the contemporaneous networks (Epskamp, Waldorp, et al., 2018). The temporal network represents the relationship between the variables over time and is estimated through a vector-autoregressive analysis (VAR; Epskamp, Waldorp, et al., 2018). When two variables are connected in the temporal network, this means that one variable predicts the other at the next time point while controlling for every other variable at the previous time point. The contemporaneous network represents relationships between the variables at the same time point, after controlling for the previous time point (temporal effects). This network takes the form of a Gaussian graphical model (GGM; Epskamp, Waldorp, et al., 2018), a network of partial correlation coefficients. When two variables are connected in the contemporaneous network, this means that the variables predict each other at the same time point while controlling for both the temporal effects and every other variable at the same time point. This modeling technique where in a VAR model the contemporaneous structure is modeled as a GGM is called the Graphical Vector Autoregressive (GVAR) model (Epskamp, Waldorp, et al., 2018; Wild et al., 2010). The GVAR model is based on three assumptions: (1) The time intervals between measurements must be approximately equal, (2) the parameters must be stationary, (3) the data must have a multivariate normal distribution (for a detailed discussion of GVAR assumptions see Epskamp, Waldorp, et al., 2018). Typically, only one lag is modelled in the GVAR, leading to a fourth assumption: (4) Any correlation between consecutive measurements beyond lag-1 correlations can be explained by lag-1 interactions. 
Different methods can be used to estimate a GVAR model (Epskamp, Waldorp, et al., 2018). In this paper, we investigate two commonly used methods in more detail. (1) The R package graphicalVAR (Epskamp, 2020a) applies regularization with penalized maximum likelihood estimation (PMLE; Abegaz \& Wit, 2013; Rothman et al., 2010). (2) The R package psychonetrics (Epskamp, 2020b) implements full information maximum likelihood (FIML) with stepwise model search (Epskamp, Rhemtulla, et al., 2017; Epskamp, 2020c). One relevant difference between PMLE and FIML is that while in PMLE all rows where at least one data point is missing are discarded, in FIML all available data points are used for estimation.

\section{Idiographic Networks and Limited Number of Observations}

Idiographic networks are regarded as a promising tool for clinical practice and research, and have already been applied in clinical settings (e.g., Frumkin et al., 2019; Kroeze et al., 2017; Robinaugh, Brown, et al., 2020). However, their use in clinical practice is not without challenges. In order to be valid tool for clinical settings, the GVAR model must successfully retrieve the most important aspects of the true network structure from clinical data, which are characterized by limited numbers of observations and the presence of missing data.

Multiple reasons contribute to the limited number of observations. First, collecting EMA data for long periods of time and at high sampling frequency may be burdensome for participants (e.g., McLean et al., 2017; Ono et al., 2019; Rintala et al., 2019; Wen et al., 2017). Second, increasing measurement frequency does not necessarily provide useful information, as this depends on the timescale of the construct of interest; for example, mood is not expected to vary every 30 minutes (Wilhelm \& Schoebi, 2007). Third, if we aim to collect data before the start of psychotherapy to develop personalized treatment plans, extending the measurement period may not be feasible, as it would cause a delay in therapy. 
Fourth, increasing the data collection period to more than a couple of weeks is undesirable, as it may make the assumption of stationarity unlikely. When estimating cross-sectional networks, sample sizes are usually in the order of hundreds to thousands. Comparable sample sizes are occasionally possible in a personalized clinical setting, and can lead to interesting results such as the predictor of a shift from a healthy to a depressed state (e.g., Wichers et al., 2016). However, collecting such rich datasets is often not feasible for idiographic networks, where $N=1,000$ would mean, for example, that one subject collected data 7 times a day for more than 5 months. The limited number of observations is especially troublesome for ideographic networks as responses are also auto-correlated (reducing effective sample size) and the number of parameters is much more due to the added temporal effects. In light of these considerations, it is essential to know the minimum sample size needed to obtain reliable results. However, no previous study investigated the performance of the GVAR model with different sample sizes in a clinical context, thus the feasibility of idiographic networks in clinical settings is unknown. The first goal of this study is to investigate whether it is possible to estimate reliable idiographic networks with sample sizes feasible in clinical settings. To this aim, we tested the performance of the GVAR model with different numbers of observations (i.e., 75, 100, 500 and 1000 time points).

\section{Idiographic Networks and Missing Data}

Missing data are very common in EMA research (e.g., McLean et al., 2017). Different factors, such as length of data collection period, sampling frequency, assessment timing, age, gender, physical activity and substance use disorder were shown to influence compliance to EMA protocols (e.g., Jones et al., 2019; McLean et al., 2017; Ono et al., 2019; Rintala et al., 2019; Wen et al., 2017). This makes it difficult to know whether data are missing completely at random (MCAR), at random (MAR), or not at random (MNAR). Common methods to handle missing data can be used with MCAR and MAR data, but they may bias the results 
with MNAR data (Graham, 2009; Shin et al., 2017). In order to solve this problem, we can use planned missingness during data collection (Graham et al., 2001, 2006) which means that we can omit some of the items at each measurement and create MCAR or MAR data that mimic item nonresponse. This is expected to reduce non-planned missingness, i.e., possible MNAR data, by reducing participant burden (Graham et al., 2001, 2006). We can then use common approaches based on data imputation or maximum likelihood estimation to deal with MCAR and MAR data (Graham, 2009). Maximum likelihood (such as FIML) is preferable to deletion techniques with MAR and MCAR data (Enders \& Bandalos, 2001; Enders, 2001) and was shown to perform better than (Enders \& Bandalos, 2001; Larsen, 2011; Shin et al., 2017; Yuan et al., 2012) or comparably to (Collins et al., 2001) imputation techniques. The Kalman filter is an imputation method for time-series data which was introduced to predict the future values of a state-space model, and was proposed as a method to address missingness in diary data (Hamaker \& Grasman, 2012; Harvey, 1989; Moritz \& Bartz-Beielstein, 2017). These approaches hold promise regarding how to deal with missing data in idiographic networks. However, no previous study investigated the performance of GVAR with missing data and different methods to address them. Thus, the second aim of the current study is to provide recommendations regarding missing data handling. To this aim, we tested the performance of GVAR with different estimation methods combined with different methods to address missing data, that is, PMLE with Kalman filter imputation (Kalman-PMLE) and stepwise model search with FIML (MS-FIML); and with different percentages of MCAR data (i.e., $0 \%, 10 \%, 25 \%$, and $50 \%$ ) characterized by item missingness.

\section{Key Questions}

To summarize, the goal of the present study is to provide recommendations to estimate reliable idiographic networks in clinical settings with regards to (1) sample size and 
(2) missing data. To this aim, we performed a simulation study to test the performance of the GVAR model with (1) different sample sizes (i.e., 75, 100, 500, 1000), (2) different amounts of missing data (i.e., $0 \%, 10 \%, 25 \%$, and $50 \%$ ), (3) different estimation methods combined with different missing data handling techniques (i.e., LASSO regularization with Kalman filter imputation, and stepwise model search with FIML). To ensure the generalizability of our results to clinical settings, we used four different true network structures estimated from two psychological datasets measuring behaviors and moods.

\section{Method}

To run the simulations, we (1) analyzed two Ecological Momentary Assessment datasets to obtain different temporal and contemporaneous network structures, (2) simulated data under these network structures, which we will call "true network structures", (3) estimated the GVAR model from these data with different sample sizes, different amounts of missing data, and different estimation methods combined with different missing data handling techniques, and (4) tested the performance of the GVAR model by comparing the estimated networks to the data-generating structures. Ethical approval for this study was obtained from the Ethics Review Board of the University of Amsterdam.

\section{Datasets}

To obtain different true network structures to simulate data from, we used two Ecological Momentary Assessment datasets where multiple individuals were measured multiple times. We chose two datasets measuring different emotions and behaviors in different samples in order to generalize our results.

\section{Dataset 1}

The first dataset was described in Piasecki et al. (2011). The sample consists of 404 current drinkers who confirmed having consumed alcohol on four or more episodes in the past 30 days. Out of 404 participants, 145 did not smoke, while 259 reported to smoke at 
least one cigarette per week. To measure EMA variables, participants used electronic diaries for 21 days.

The EMA protocol included five types of self-report assessments: (a) time-based random prompts, (b) user-initiated cigarette reports in which subjects indicated occurrences of cigarette smoking, (c) user-initiated drinking reports in which participants indicated occurrences of the first drink in a drinking episode, (d) a series of automated drinking followups collected after each user-initiated drinking report, (e) morning assessments completed every day after waking up. For the current analysis we only used random prompts, because incorporating different kinds of entries in the same model would make networks' interpretation arduous.

Random prompts were delivered for a maximum of five times each day. If subjects could not fill out the items, they were allowed to reschedule the measurement up to 20 minutes later. Random prompts scheduled after the user-initiated drinking reports were not delivered due to the automated drinking follow-ups. The full set of EMA variables was described in Piasecki et al. (2011). After excluding categorical variables and variables with more than $80 \%$ missing values, the following EMA variables were included (see Table 1): (a) positive affect variables: enthusiastic, excited, happy; (b) negative affect variables: distressed, sad; (c) craving: crave a cigarette, crave a drink; (d) effects of alcohol intoxication and alcohol withdrawal/hangover: buzzed, dizzy, sluggish, headache, nauseous. These subjective states were rated on a 5 -point scale from $1=$ not at all to $5=$ extremely, and participants were asked to answer taking into account the past 15 minutes.

\section{Dataset 2}

The second dataset was described in Fried et al. (2020). The sample consists of 79 bachelor students of the University of Leiden. Participants collected EMA variables for 2 
weeks from March 16 to March 292020 during the beginning of the first lockdown measures due to the COVID-19 crisis (University shut down on March 13).

EMA variables were collected four times per day (12 am, 3 pm, 6 pm, 9 pm) through smartphones. Participants had 1 hour to respond before the assessment became unavailable. The complete data collection procedures were reported in Fried et al. (2020). For the current study, we included 14 EMA variables (see Table 1) investigating how much participants perceived a specific feeling (not at all, slightly, moderately, very, extremely), or how much time they dedicated to a specific activity (0 minutes, 1-15 minutes, 15-60 minutes, 1-2 hours, over 2 hours). Participants were asked to respond taking into account the last 3 hours.

\section{Table 1}

\section{EMA Items of the Two Datasets}

\begin{tabular}{|c|c|}
\hline Abbreviation & Item \\
\hline \multicolumn{2}{|r|}{ Dataset 1} \\
\hline Enthusiastic & In the PAST 15 MINUTES, did you feel ENTHUSIASTIC? \\
\hline Excited & In the PAST 15 MINUTES, did you feel EXCITED? \\
\hline Happy & In the PAST 15 MINUTES, did you feel HAPPY? \\
\hline Distressed & In the PAST 15 MINUTES, did you feel DISTRESSED? \\
\hline Sad & In the PAST 15 MINUTES, did you feel SAD? \\
\hline Crave Cigarette & In the PAST 15 MINUTES, did you CRAVE A CIGARETTE? \\
\hline Crave Drink & In the PAST 15 MINUTES, did you CRAVE A DRINK? \\
\hline Buzzed & In the PAST 15 MINUTES, did you feel BUZZED? \\
\hline Dizzy & In the PAST 15 MINUTES, did you feel DIZZY? \\
\hline Sluggish & In the PAST 15 MINUTES, did you feel SLUGGISH? \\
\hline Headache & In the PAST 15 MINUTES, did you feel HEADACHE? \\
\hline Nauseous & In the PAST 15 MINUTES, did you feel NAUSEOUS? \\
\hline \multicolumn{2}{|r|}{ Dataset 2} \\
\hline Difficult Relax & I found it difficult to relax \\
\hline Irritable & I felt (very) irritable \\
\hline Worry & I was worried about different things \\
\hline Nervous & I felt nervous, anxious or on edge \\
\hline Future & I felt that I had nothing to look forward \\
\hline Anhedonia & I couldn't seem to experience any positive feeling at all \\
\hline Tired & I felt tired \\
\hline
\end{tabular}




\begin{tabular}{ll}
\hline Alone & I felt like I lack companionship, or that I am not close to people \\
Social Offline & I spent__ minutes/hours on meaningful, offline, social interaction \\
Social Online & I spent__ minutes/hours using social media to kill/pass the time \\
Outdoors & I spent__ minutes/hours outside (outdoors) \\
C19 Occupied & $\begin{array}{l}\text { I spent_ minutes/hours occupied with the coronavirus (e.g. watching } \\
\text { news thinking about it, talking to friends about it) }\end{array}$ \\
C19 Worry & $\begin{array}{l}\text { I spent__minutes/hours thinking about my own health or that of my } \\
\text { close friends and family members regarding the coronavirus }\end{array}$ \\
Home & I spent_minutes/hours at home (including the home of parents/partner) \\
\hline
\end{tabular}

Note. Part of this table was adapted from "Mental health and social contact during the

COVID-19 pandemic: an ecological momentary assessment study,” by E. I. Fried, F.

Papanikolaou, and S. Epskamp, 2020, PsyArXiv (https://doi.org/10.31234/osf.io/36xkp).

\section{Statistical Analysis}

All statistical analyses were conducted with the statistical software $\mathrm{R}$ version 4.0.2. The syntax for the analyses of Dataset 2 is available in the Supplementary Materials, as Dataset 2 is freely available online for replication. Dataset 1 is not available for replication, but the analyses were largely the same as those for Dataset 2 . When small differences were present, this was explained the paper.

\section{Obtaining the true network structures}

Two-step multilevel vector autoregression (two-step mlVAR; Epskamp, Waldorp, et al., 2018) was run with the R package $m l V A R$ (Epskamp, Deserno et al., 2019) to obtain the lag-1 average within-subject temporal networks. Residuals were modeled in a second step to obtain the average within-subject contemporaneous networks. These temporal and contemporaneous networks were used as the true networks to simulate data from.

Since two-step mlVAR assumes stationarity, Dataset 2 was detrended (Fried et al., 2020). Fixed-effects linear regressions were fitted to every variable included in the network. 
The number of the day and the number of the measurement in the day were used as predictors and regressed out at a significance level of 0.05 . The same procedure was carried out for Dataset 1, but in this case only the linear trend of day number was regressed out. The effect of measurement per day was not regressed because prompts were delivered at random, therefore time-intervals between two measurements were variable.

Dynamical networks were estimated with different numbers of nodes $(12,8$, and 6 nodes for Dataset 1; 9 nodes for Dataset 2) to test whether GVAR performance would improve with less parameters to be determined. To estimate a network structure with 8 nodes for Dataset 1, we averaged the items Enthusiastic, Excited, and Happy to obtain the variable Positive Affect; Buzzed and Dizzy to obtain the variable Physical Substances; and the items Headache and Nauseous to obtain the variable Physical Generic. This decision was based on the assumption that feeling buzzed and dizzy may be perceived as a pleasurable consequence of alcohol and tobacco use (e.g., DiFranza et al., 2004; C. S. Pomerleau et al., 1999; O. F. Pomerleau et al., 1998), while having a headache or feeling nauseous are not. To estimate a network structure with 6 nodes for Dataset 1 we averaged the items Distressed and Sad to obtain the variable Negative Affect; and the items Physical Substances and Physical Generic to obtain the variable Physical. To estimate a network structure with nine nodes for Dataset 2 we averaged the items Future, Anhedonia, and Tired to obtain the variable Depression; and the items Difficult Relax, Irritable, Worry, and Nervous to obtain the variable Anxiety. These sum-scores were based on the Diagnostic and Statistical Manual of Mental Disorders (DSM) 5, which indicates anhedonia, feeling tired and hopeless as central symptoms of Major Depressive Disorder (MDD), while worrying, feeling anxious, irritable and restless as central symptoms of Generalized Anxiety Disorder (GAD).

\section{Simulations}


The simulations were set up using the R package parSim (Epskamp, 2019). The function graphicalVARsim from the R package graphicalVAR was used to simulate the data from the true models. The data were simulated under different conditions: with $0 \%, 10 \%$, $25 \%$, and $50 \%$ of item missingness completely at random, and with a number of time points of $75,100,500$, and 1000 . These time points were chosen to represent typical sample sizes in $N=1$ studies $(75,100)$ and very large sample sizes in $N=1$ studies occasionally seen in rich datasets $(500,1000)$. The dynamical networks were then estimated from the simulated data using either MS-FIML (with the R package psychonetrics) or Kalman-PMLE (with the R package graphicalVAR). In psychonetrics the model was first pruned by removing nonsignificant edges at alpha $=0.01$, then stepwise model search was performed with prunealpha (the minimum alpha to consider removing edges) $=0.01$ and add-alpha (the maximum alpha to consider adding edges $)=0.01$ until the Bayesian Information Criterion $(\mathrm{BIC}$; Neath \& Cavanaugh, 2012) could no longer be optimized (Epskamp, 2020c). In graphicalVAR we set gamma, the Extended BIC (EBIC; J. Chen \& Z. Chen, 2008; Foygel \& Drton, 2010) hyperparameter, to 0 . Consequently, regular BIC was used which is slightly less conservative and is expected to improve sensitivity. Before estimating the model with graphicalVAR, we applied Kalman filter imputation with the R package imputeTS (Moritz \& Bartz-Beielstein, 2017). Since FIML uses all available information during estimation, no additional method to deal with missingness was needed. The total number of simulation conditions was 32 $(4 \times 4 \times 2)$. Each condition was repeated 100 times, leading to a total of 32.000 simulated datasets for each true network structure.

\section{Network Estimation Performance}

To investigate the performance of the GVAR model in the different conditions, we calculated the specificity, sensitivity, bias, precision, and the correlation between estimated and true edges (as previously done, e.g., de Ron et al., 2019; Epskamp, 2020c; Epskamp, 
Waldorp, et al., 2018; Epskamp \& Fried, 2018). Specificity is the true-negative rate, that is, the proportion of edges that were missing in both the true and estimated networks. Sensitivity is the true-positive rate, that is, the proportion of edges that had nonzero values in both the true and estimated networks. The bias is the mean absolute deviation of estimated edges from the true edges. The correlation quantifies the similarity between the edge weights of the true and estimated networks. When the estimated network was empty, it was not possible to calculate the correlation, therefore the proportion of estimated empty networks is available in the Supplementary Materials. Precision is the proportion of detected edges that are also true. Consequently, network estimation performs better if the specificity, sensitivity, precision and correlation are large, while bias is small. Additionally, we estimated sensitivity for the strongest $25 \%$ and $50 \%$ edges (proportion of times these edges were included in the estimated network).

\section{Results}

\section{Dataset 1}

\section{Estimated network structures}

Figures 1, 2 and 3 show, respectively, the true networks estimated from Dataset 1 with 12, 8, and 6 nodes. To better compare the networks, edges width and saturation were scaled based on the maximum connection found across all networks in Dataset 1 (i.e., $r=0.43$ ). Empirical results should be taken with caution, as Dataset 1 violates the assumption of equality of the time intervals. However, the networks seem to be in line with what we would expect based on theory and previous research, and give an idea of what a substance use network may look like in a clinical setting.

In the contemporaneous network with 12 nodes, feeling happy, excited and enthusiastic are positively related. The same is true for feeling distressed and sad, which are also negatively related to feeling happy. Craving a cigarette is positively related to craving a 
drink, suggesting that if someone craves a drink, he/she is also more likely to crave a cigarette at the same time. Physical symptoms are positively related with each other and exhibit different relationships with negative and positive affect. Feeling sluggish is negatively related to positive affect items and positively related to negative affect items. Having a headache and feeling nauseous are positively related with negative affect items. Feeling buzzed was previously suggested to be pleasurable (DiFranza et al., 2004; C. S. Pomerleau et al., 1999; O. F. Pomerleau et al., 1998), and is indeed positively related to positive affect items. Feeling buzzed is also positively related to craving a drink or a cigarette, while feeling sluggish is negatively related to craving a cigarette. In line with previous research, both negative and positive affect items are positively related with craving (Serre et al., 2015).

In the temporal network with 12 nodes, positive affect items positively predict each other over time. The same is true for negative affect items. As expected, positive and negative affect negatively predict each other over time. Craving a drink and a cigarette also predict each other over time, meaning that if someone craves a drink at time point one, he/she is also more likely to crave a cigarette at time point two and vice versa. Physical symptoms positively predict each other over time and, again, exhibit different relationships with other variables. Feeling nauseous negatively predicts craving a cigarette and feeling sluggish negatively predicts craving both a cigarette and a drink. Craving a drink predicts feeling buzzed, dizzy, excited and enthusiastic at the next time point. It is arduous to interpret this connection, as we do not know whether people actually had a drink or a cigarette when craving one. Finally, feeling sad positively predicts craving a cigarette. Here we do not see other predictions going from positive and negative affect to craving.

The network with 8 nodes largely replicates the results with 12 nodes. The network with 6 nodes replicates the results with 12 nodes with one exception: Here we calculated the sum score of having a headache, feeling nauseous, buzzed, and dizzy and called this variable 
"physical". This variable is now positively related to both positive and negative affect, and the difference between the effect of feeling buzzed and feeling nauseous or having a headache is not distinguishable.

\section{Simulation results}

Figures 4, 5 and 6 show, respectively, the results of the simulations for the networks estimated from Dataset 1 with 12, 8, and 6 nodes. For the sake of clarity, results are shown for the full model (contemporaneous and temporal networks together). The Supplementary Materials contain the separate results for the two kinds of networks, the complete descriptive statistics, the proportion of failed estimations (i.e., the cases where no results could be reached due to numerical optimization problems), and the proportion of estimated empty networks.

Our first question regards sample size. Here the most striking result is that sensitivity is small, especially with a number of time points that is feasible for clinical practice $(N=75$, $N=100$ ); this means that power is small, and false negatives are likely to occur. Sensitivity improves when estimating a network with a smaller number of nodes, when the number of time points increases, when using the graphicalVAR package compared to psychonetrics, and when the percentage of missing data decreases; however, it remains small with $N=75$ and $N$ $=100$. For example, sensitivity with 6 nodes, $0 \%$ missing data and graphicalVAR has $M d n=$ $0.19, M=0.20, S D=0.08$ with $N=75$, and $M d n=0.23, M=0.24, S D=0.09$ with $N=100$. The true networks are dense and feature many weak edges, which is expected to reduce sensitivity because (1) the regularization and model selection techniques used during the estimation process were developed to estimate sparse networks (Epskamp, Kruis, et al., 2017), (2) a larger power is needed to detect small effects. As sensitivity is based on the presence or absence of an edge, and does not consider edge weight, sensitivity for the strongest $25 \%$ and $50 \%$ edges is particularly informative. This is larger than general 
sensitivity, indicating that strongest edges are more likely to be detected. The correlation between the true and estimated network parameters, which is also relevant as it takes into account edge weight, increases with increasing sample sizes and decreasing percentages of missing data. In general, the values for the correlation seem to be fairly good, also for small sample sizes (especially with $N=100$ ). For example, with six nodes, $0 \%$ missing data and graphicalVAR, the correlation has $M d n=0.53, M=0.54, S D=0.12$ with $N=75$ and $M d n=$ $0.64, M=0.63, S D=0.11$ with $N=100$. This indicates that the true and estimated networks tend to have a similar global structure, but, due to small power, we cannot expect to retrieve the full network.

Interestingly, results are slightly different for the temporal and contemporaneous networks (see Supplementary Materials, Figures 1,2,3,4,5,6). Estimation seems to perform better for the contemporaneous network, as indicated by a larger correlation between the true and estimated network parameters. For example, with $0 \%$ missing data, graphicalVAR and $N$ $=100$, the correlation for the contemporaneous network has $M d n=0.73, M=0.69, S D=0.16$ with 6 nodes and $M d n=0.74, M=0.75, S D=0.06$ with 12 nodes. In the same conditions, the correlation for the temporal network has $M d n=0.67, M=0.64, S D=0.18$ with 6 nodes and $M d n=0.51, M=0.49, S D=0.09$ with 12 nodes. This also shows that the number of nodes has a larger impact on the estimation of the temporal compared to the contemporaneous network, and keeping the number of nodes low is particularly important to estimate the former.

Our second question regards missing data. Specificity is always large and bias always small, indicating that the estimated edges are unlikely to be false positives, and both FIML and the Kalman filter are effective in addressing missing data. However, precision and correlation diminish with increasing percentages of missing data, thus the larger the percentage of missing data, the more likely some of the estimated edges will be false 
positives. This is troublesome when the effective sample size is really low, such as with $N=$ 75 and proportion of missing data $=0.5$. In this case, psychonetrics only yields failed estimations, while graphicalVAR performance tends to decline. This means that planned missingness is a promising method to collect more data points while reducing participants' burden, but the number of data points available should not be too low. 


\section{Figure 1}

Contemporaneous and Temporal Networks for Dataset 1 with 12 Nodes
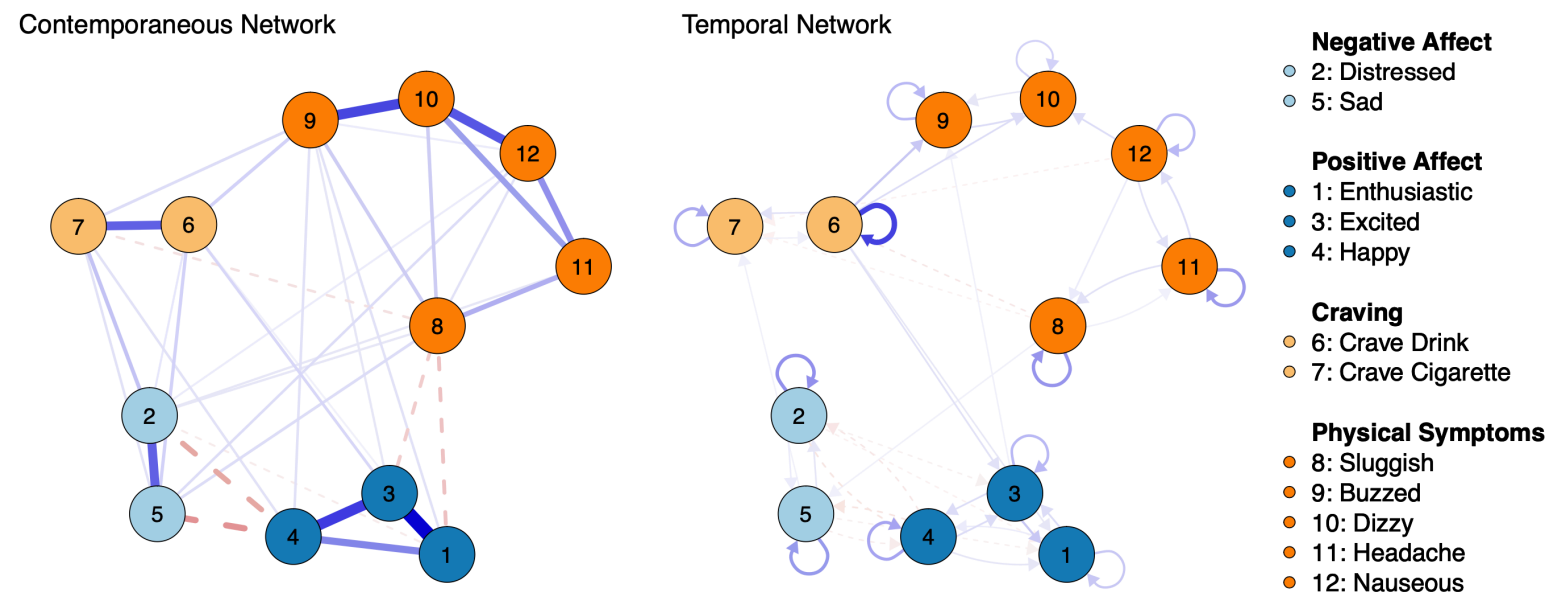

Note. Dashed red edges represent negative partial correlations, while solid blue edges represent positive partial correlations. Edge width and saturation were scaled based on the maximum edge found across all networks estimated with Dataset $1(r=0.43)$, thus wider and brighter edges indicate stronger connections. 


\section{Figure 2}

Contemporaneous and Temporal Networks for Dataset 1 with 8 Nodes
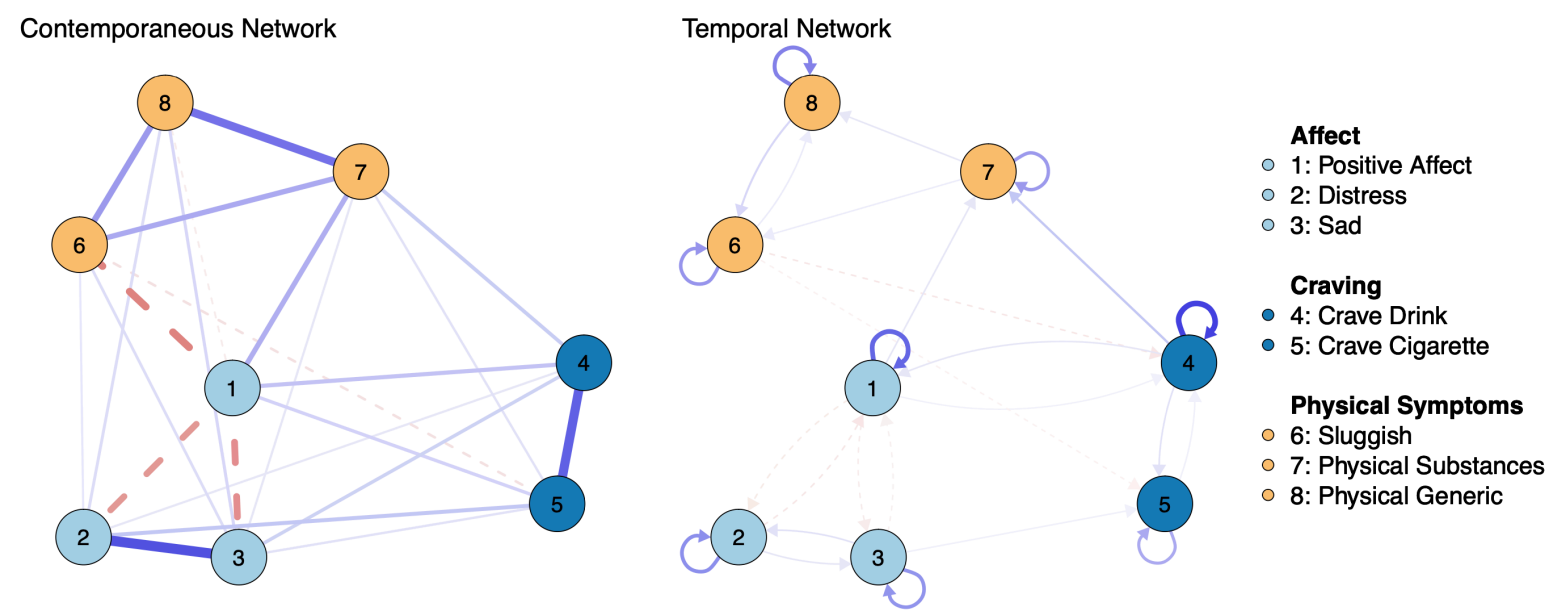

Note. Dashed red edges represent negative partial correlations, while solid blue edges

represent positive partial correlations. Edge width and saturation were scaled based on the maximum edge found across all networks estimated with Dataset $1(r=0.43)$, thus wider and brighter edges indicate stronger connections. 


\section{Figure 3}

Contemporaneous and Temporal Networks for Dataset 1 with 6 Nodes

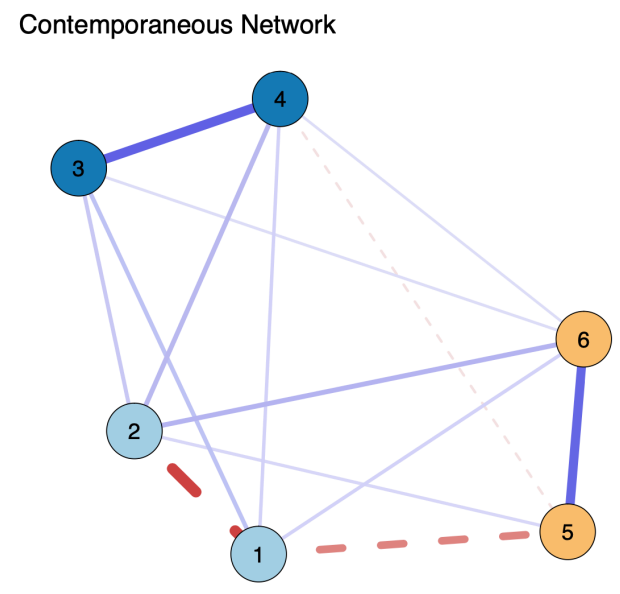

Temporal Network
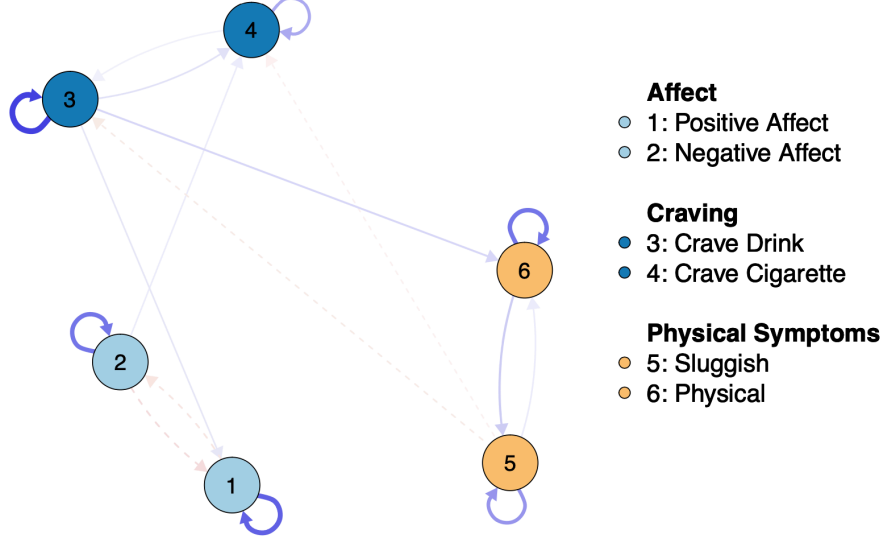

Note. Dashed red edges represent negative partial correlations, while solid blue edges

represent positive partial correlations. Edge width and saturation were scaled based on the maximum edge found across all networks estimated with Dataset $1(r=0.43)$, thus wider and brighter edges indicate stronger connections. 


\section{Figure 4}

Simulation Results for the Network Estimated from Dataset 1 with 12 Nodes

— GraphicalVAR With Kalman — Psychonetrics With FIML and Model Search

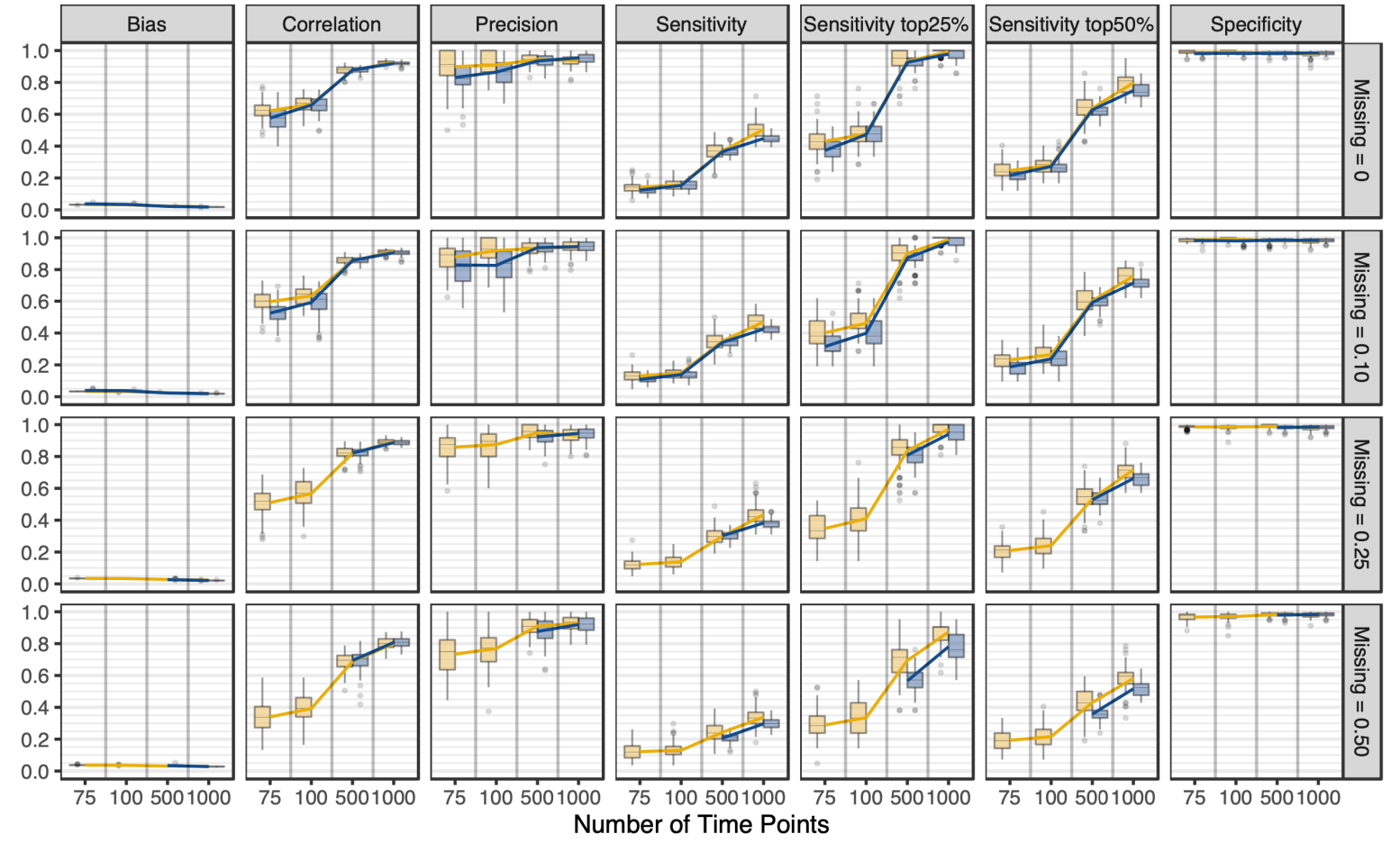

Note. The upper and lower bounds of the boxplot represent the 75 th and 25 th percentiles. The upper whisker reaches the maximum value within $1.5^{*}$ the interquartile range (IQR) from the upper bound. The lower whisker reaches the minimum value within $1.5 *$ IQR from the lower bound. Outliers that exceed the whiskers are represented as points. The lines plot the means in each different condition. 


\section{Figure 5}

Simulation Results for the Network Estimated from Dataset 1 with 8 Nodes

— GraphicalVAR With Kalman — Psychonetrics With FIML and Model Search
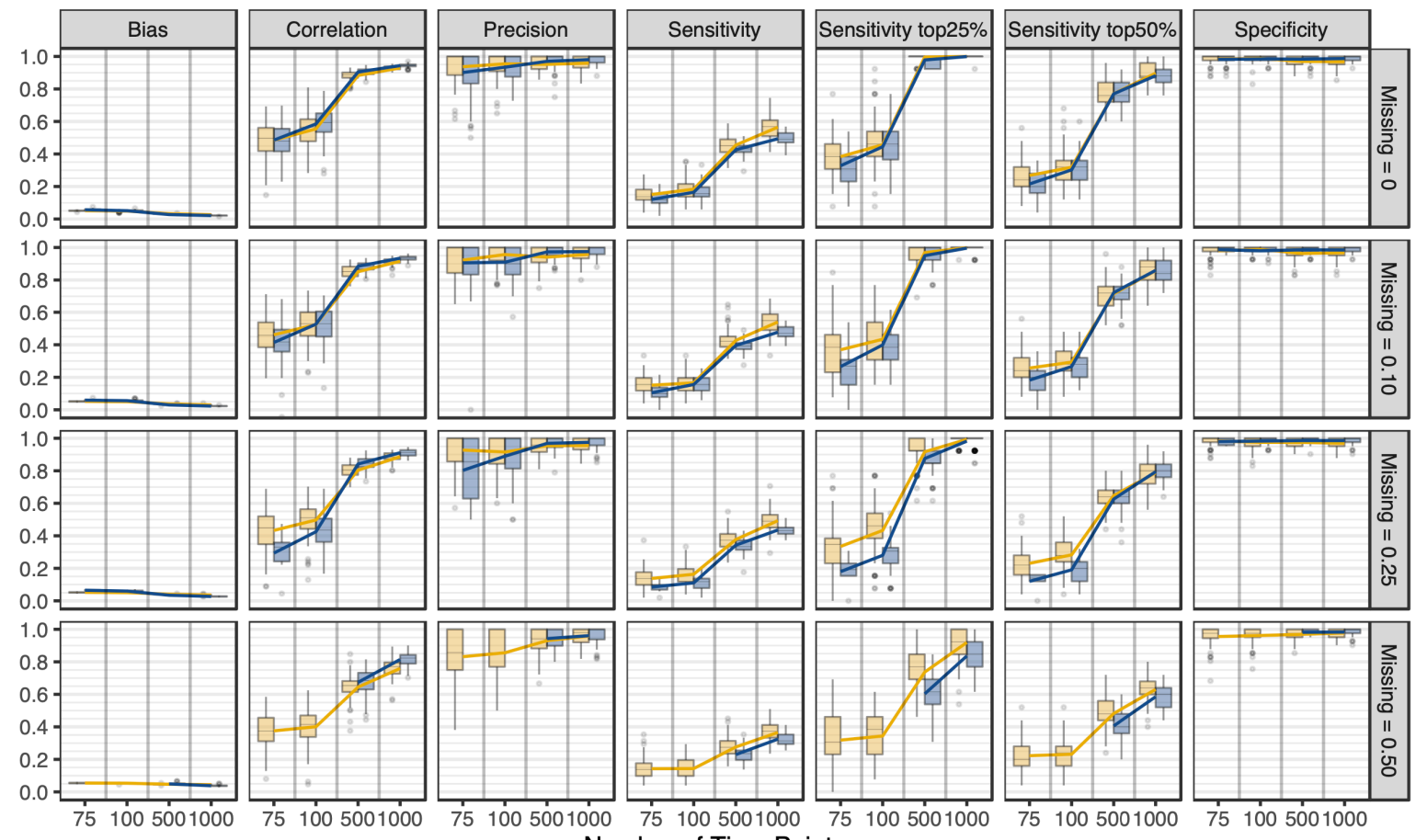

Number of Time Points

Note. The upper and lower bounds of the boxplot represent the 75th and 25th percentiles. The upper whisker reaches the maximum value within $1.5^{*}$ the interquartile range (IQR) from the upper bound. The lower whisker reaches the minimum value within $1.5 *$ IQR from the lower bound. Outliers that exceed the whiskers are represented as points. The lines plot the means in each different condition. ${ }^{1}$

\footnotetext{
${ }^{1}$ One outlier correlation $(r=-0.044)$ between the true and estimated networks was not plotted, but it was included in the summary statistics. This was found with $\mathrm{N}=75$, proportion of missing data $=0.10$, and psychonetrics. A negative correlation between the true and estimated networks can be found in cases where estimation performs poorly.
} 


\section{Figure 6}

Simulation Results for the Network Estimated from Dataset 1 with 6 Nodes

- GraphicalVAR With Kalman — Psychonetrics With FIML and Model Search
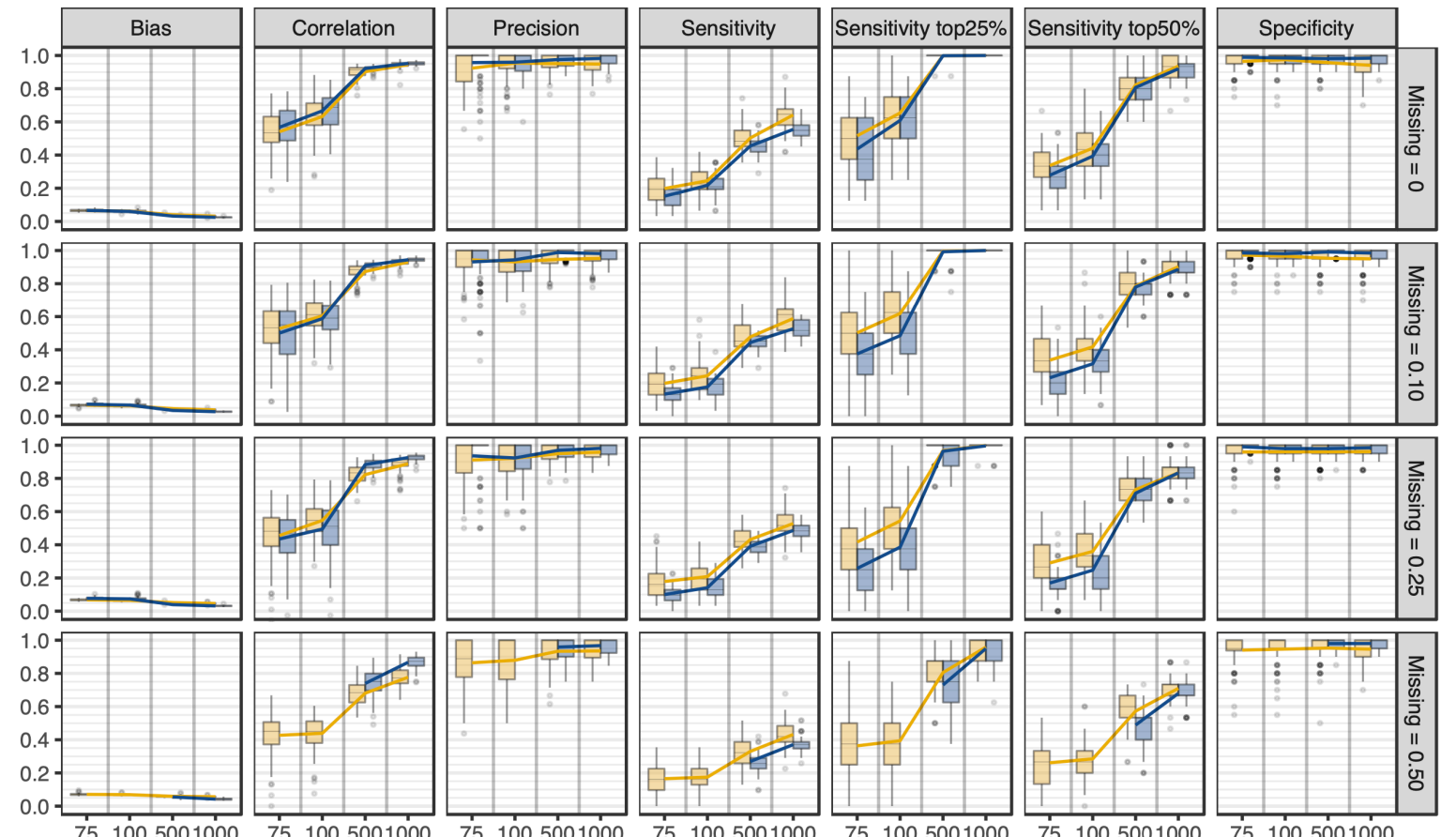

Number of Time Points

Note. The upper and lower bounds of the boxplot represent the 75 th and 25 th percentiles. The upper whisker reaches the maximum value within $1.5^{*}$ the interquartile range (IQR) from the upper bound. The lower whisker reaches the minimum value within $1.5 *$ IQR from the lower bound. Outliers that exceed the whiskers are represented as points. The lines plot the means in each different condition. ${ }^{2}$

\section{Dataset 2}

\section{The Networks}

Figure 7 shows the true networks estimated from Dataset 2 with 9 nodes. To better compare the contemporaneous and temporal networks, edge width and saturation were scaled

\footnotetext{
${ }^{2}$ Three outlier correlations between the true and estimated networks ranging from -0.056 to -0.026 were not plotted, but they were included in the summary statistics. These were found with $\mathrm{N}=75$ and $\mathrm{N}=100$ and proportion of missing data $=0.25$.
} 
based on the maximum edge found across these two $(r=0.51)$. The networks for this dataset were described in Fried et al. (2020), where they were estimated including all mental health items separately (i.e., items were not aggregated to create the nodes Depression and Anxiety and the total number of nodes was 14). In our study, we only used 9 nodes because the 14 nodes networks were expected to be too complex to be estimated with the data of one subject alone. The networks with 9 nodes replicate the main results of the original 14 nodes networks, with the difference that in the original networks more details were available for specific mental health items, as these were separately represented. For example, feeling nervous predicted feeling less alone at the next time point, which is not observable in the 9 nodes network.

\section{The Simulation}

Figure 8 shows the simulation results for these networks. In general, the results for Dataset 2 are in line with and confirm the results for Dataset 1. Interestingly, here the difference in estimation performance between the contemporaneous and temporal networks is even stronger (see Supplementary Materials, Figures 7 and 8). Correlation with $0 \%$ missing data, graphicalVAR, and $N=100$ has $M d n=0.88, M=0.88, S D=0.04$ for the contemporaneous network; and $M d n=0.35, M=0.34, S D=0.17$ for the temporal network. 


\section{Figure 7}

Contemporaneous and Temporal Networks for Dataset 2 with 9 Nodes
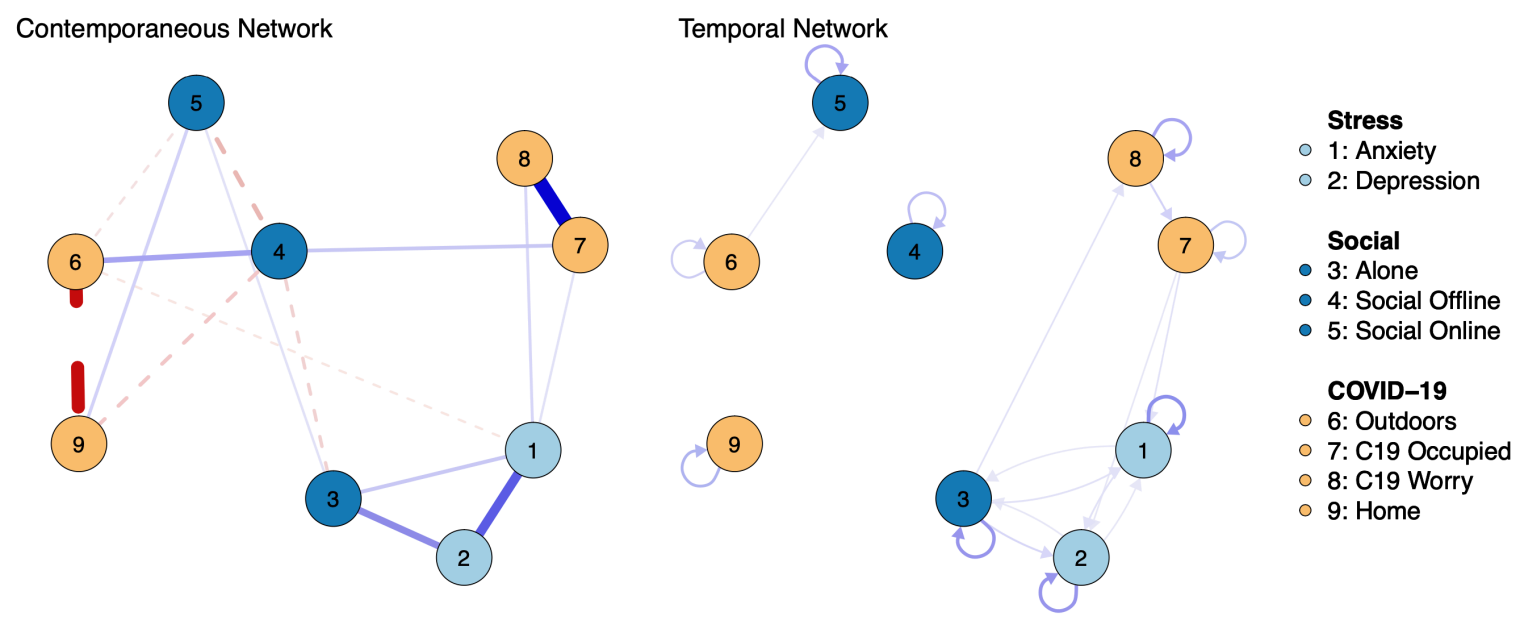

Note. Dashed red edges represent negative partial correlations, while solid blue edges represent positive partial correlations. Edge width and saturation were scaled based on the maximum edge found across the temporal and contemporaneous networks estimated with Dataset $2(r=0.51)$, thus wider and brighter edges indicate stronger connections. Adapted from "Mental health and social contact during the COVID-19 pandemic: an ecological momentary assessment study," by E. I. Fried, F. Papanikolaou, and S. Epskamp, 2020, PsyArXiv (https://doi.org/10.31234/osf.io/36xkp). 


\section{Figure 8}

Simulation Results for the Network Estimated from Dataset 2 with 9 Nodes

— GraphicalVAR With Kalman — Psychonetrics With FIML and Model Search
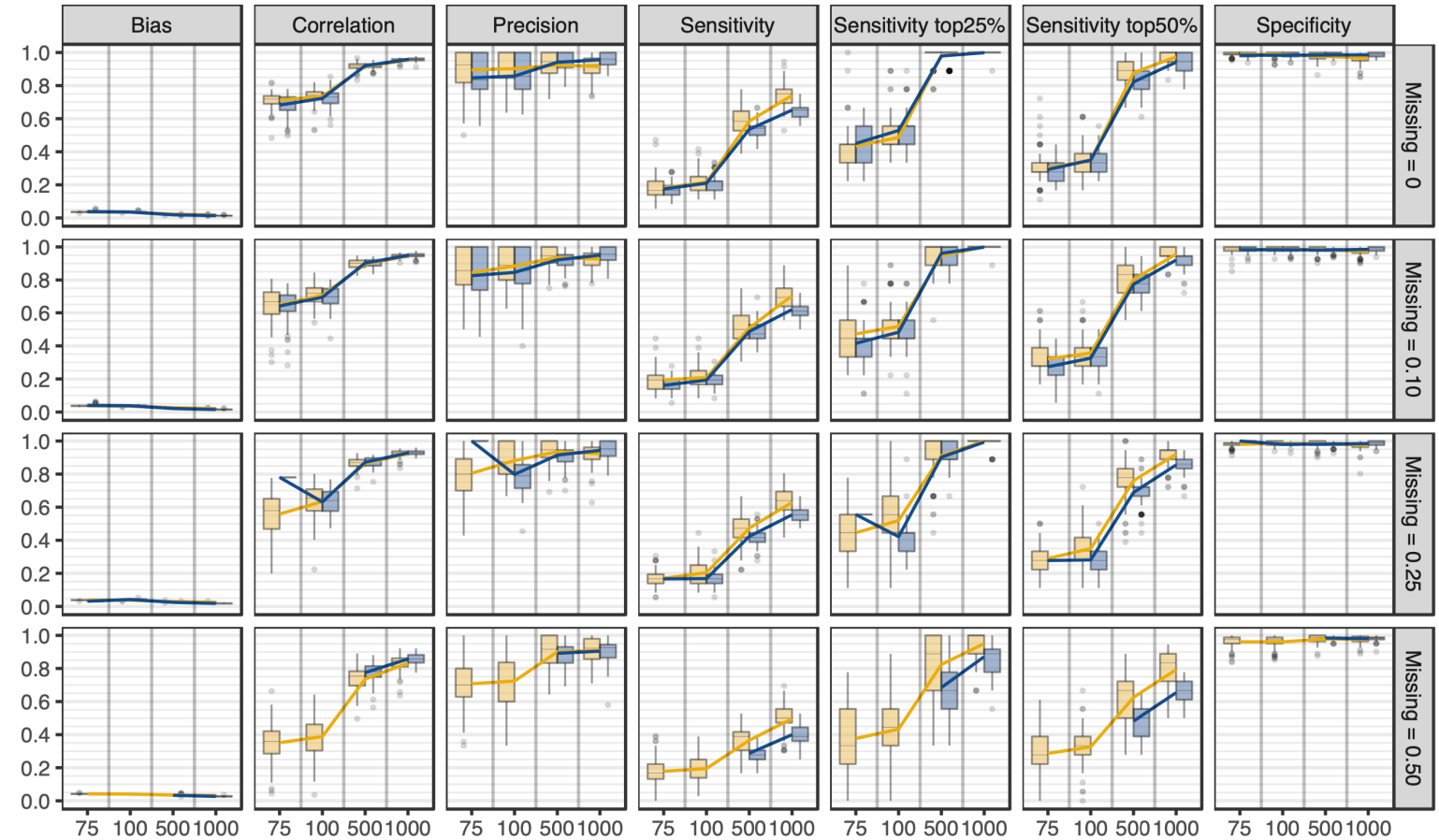

Number of Time Points

Note. The upper and lower bounds of the boxplot represent the 75 th and 25 th percentiles. The upper whisker reaches the maximum value within $1.5^{*}$ the interquartile range (IQR) from the upper bound. The lower whisker reaches the minimum value within $1.5 *$ IQR from the lower bound. Outliers that exceed the whiskers are represented as points. The lines plot the means in each different condition.

\section{Discussion}

\section{Summary and Methodological Recommendations}

The goal of the present study was to provide recommendations for the application of personalized networks in clinical research and practice. Specifically, we investigated (1) whether it is possible to estimate reliable idiographic networks with numbers of observations feasible in clinical settings and (2) how to handle missing data. 
Regarding number of observations, the most striking result was low sensitivity, especially with a number of measurements feasible in clinical practice $(N=75$ and 100). At the same time, the correlation between the true and estimated networks was in an acceptable range. This indicates that the full network cannot be obtained, but the global structure of the network is likely to be recovered. Sparse networks were previously observed in the literature (e.g., Frumkin et al., 2019). According to our results, sparsity is likely to be caused by low power and should not be interpreted as an actual lack of connections. To increase sensitivity, different recommendations can be followed. First, as many data points as possible should be collected. In case longer measurement periods cause nonstationary, the data should be detrended and the trends interpreted. Second, the number of nodes should be reduced as much as possible. To reduce the number of nodes, in the current study we aggregated variables, which was generally successful considering that networks with different numbers of nodes were similar. However, the results from Dataset 1 showed that when symptoms representing different factors are aggregated (physical symptoms in the 6 nodes network), the relationship with other variables may become unclear (here with affect). Therefore, item aggregation should be based on theory, factor analysis, or previous research. Another approach would be to use latent network models (Epskamp, 2020c) to aggregate nodes directly in the idiographic networks. Third, LASSO regularization with Kalman filter imputation (grapicalVAR) seems to be slightly more powerful than model search with FIML (psychonetrics) and should be preferred with small sample sizes.

GVAR performed worse when retrieving the temporal versus the contemporaneous networks, and the number of nodes had a stronger impact on the estimation of the former. This difference could be explained by the fact that the true contemporaneous networks contained stronger connections compared to the temporal ones, which may be a common characteristic of networks in clinical settings. However, a similar effect was also visible in 
previous simulations with synthetic true networks (Epskamp, Waldorp, et al., 2018), where no difference between the structure of temporal and contemporaneous networks was present. According to our results, 6 seems to be a reasonable number of nodes to recover the global temporal structure with sample sizes between 75 and 100 data points. It is also important to remember that estimation performance depends in part on the characteristics of the network structure, therefore performance may differ with different datasets. For example, in the current study retrieving the temporal network was easier in Dataset 1 compared to Dataset 2 (see Supplementary Materials). It is consequently essential to test and provide methods that can estimate the accuracy of each specific idiographic network that is being estimated. Such methods are available for cross-sectional networks (Epskamp, Borsboom, et al., 2018), and include, for example, bootstrapped confidence intervals or analytical standard errors of the edge-weights.

Regarding missing data, both FIML and the Kalman filter were effective in addressing random item-level missing data. By planning random item nonresponse, we can collect more data points while reducing participants' burden (Harel et al., 2015), which can (1) improve power and (2) diminish the probability of data missing not at random and wave nonresponse. Planned missingness can be made more efficient by implementing (network based) adaptive assessment. The distribution of some network parameters may be clear after a smaller number of measurements. Based on this, some items can be excluded from the next measurement occasions and predicted using the other items that are assessed. The results of the current study indicate that planned item nonresponse can be successfully handled, thus planned missingness and adaptive assessment are promising strategies to deal with missing data in personalized networks. As precision and correlation can decrease with larger percentages of missing data, it is important to find a balance between the percentage of planned missingness used and the number of measurements collected. With larger 
percentages of planned missing data, the number of time-points should also be larger. For example, with 100 time points and 50\% missing data, only 50 data points are available, which is likely too small to reliably retrieve even the global network structure. Consequently, it is not advisable to use large percentages of planned missingness such as $50 \%$ unless the measurement period is very long and the measurement frequency large.

Beyond opening the doors to planned missingness, the current manuscript also shows that missing data in EMA samples can be handled through both imputation and fullinformation strategies. It should be noted, however, that missing data in EMA studies usually exhibit wave nonresponse as opposed to item nonresponse (McLean et al., 2017; Schafer \& Graham, 2002), which means that participants are likely to miss all the items at a certain time point. Since in wave nonresponse all data in a wave are missing, methods that use all available information as discussed in this manuscript are not very different from listwise deletion.

\section{Future Directions}

The given recommendations alone are unlikely to solve the problem of low power. Does this mean that idiographic networks are not feasible for clinical practice? Probably not. First of all, in the current study the true networks were estimated from real datasets using multilevel analysis, which uses data from many participants and is highly powerful. This means that our true networks were representative of what we may expect in clinical settings, but also that we were able to detect many weak edges, which increase estimation difficulty. Power will improve if we try to retrieve a true network with a more defined and sparse structure, i.e., containing stronger and fewer edges. Epskamp, Waldorp and colleagues (2018) performed a simulation study with graphicalVAR and synthetically generated true networks, which have a well-defined sparse structure (see pages 11 and 13 of their Supplementary Materials). In their simulation GVAR performed much better compared to the present study. 
With 100 time points and 8 nodes, sensitivity had a mean ranging from 0.83 to 0.89 across different conditions. Sometimes, in clinical practice we may not be interested in detecting small edges, but only the strongest and most relevant edges. However, in some cases, weak edges may also be relevant, their small effect-size may be due to other factors such as small variation of the item over time or sampling bias. Imagine a patient who smokes cannabis every day multiple times a day. The variable smoking cannabis will have little variability, thus it will not exhibit strong connections with other variables in the network. However, this does not mean that smoking is not causing, for example, psychotic symptoms and a negative baseline mood. And imagine that we measure smoking cannabis and paranoia for two weeks every four hours in an individual who recently started smoking daily. We may find that smoking only weakly predicts paranoia after four hours. However, smoking every day may be strongly related to paranoia after, for example, 6 months. The time scale of most psychological processes is unclear, and EMA sampling rate may sometimes be unsuitable to detect causal links.

Fortunately, different approaches can be taken to solve the problem of small power together with low variability and sampling bias. First, the multilevel approach could be implemented in clinical practice. Multilevel analysis is powerful because it allows to estimate individual networks while also borrowing information from other clients with similar problems. This method is usually not feasible for personalized clinical practice, where we cannot wait for many clients to be tested before we start therapy for one person. However, in the future we could imagine having time-series networks estimated in a multilevel fashion from big datasets for every different disorder. These networks could be readily available to clinicians to be used together with the personalized network of the client to fill in possible edges that were not detected due to small power. Data previously collected in other patients with similar problems could also be used to estimate the personalized network in a multilevel 
fashion, shrinking individual estimates towards the fixed effects. Second, instead of using EMA data to estimate the network, a network of prior expectations could be built based on the knowledge of the therapist, the expectations of the client, theory, and results from previous research. The data could then be used to update this network with a Bayesian approach (Burger, Epskamp, van der Veen, Dablander, Schoevers, Riese \& Fried, in preparation). Third, confirmatory instead of exploratory network analysis could be performed by first creating a theoretical network based on prior expectations, and then fitting data to this model with an approach similar to structural equation modeling (SEM) as implemented in psychonetrics (Epskamp, 2020c; Epskamp, Rhemtulla, et al., 2017). Fourth, in order to deal with small variation and to increase power, one could decide whether or not to include variables in the network based on variability. Future studies could aim to identify indicators for low variability of nodes. If the variables with low variability are clinically relevant (as in the example above), they could be included in the network through different means, e.g., therapist knowledge and client expectations.

Other methodological advancements are also possible. As highlighted above, future studies should provide methods that allow researchers to estimate the accuracy of the specific idiographic network they are estimating. Additionally, so far, self-report data alone have been used to estimate personalized networks. Future research should aim to integrate in the personalized networks passive data measured with wearables, such as heart rate, global positioning system (GPS) location, and movement. Passive data would provide objective information that has two advantages compared to self-report: (1) It does not have the same validity problems of EMA items, (2) it requires less effort from participants.

\section{Considerations for the Application of Idiographic Networks in Psychotherapy}

Given that we aimed to test GVAR feasibility for clinical practice, a question arises: What are the consequences of our results for the application of idiographic networks in 
psychotherapy? Methods that integrate therapist and patient expectations into the personalized network not only have the potential to help overcoming GVAR limitations, but also to foster the communication between patient and therapist, improve the discussion about the psychological mechanisms of the client, actively involve the client into the therapeutic process, and motivate the client and the therapist to use new idiographic methods. Idiographic networks could be implemented in clinical practice in a collaborative fashion (Spencer et al., 2019; Tryon et al., 2018). Before the start of psychotherapy, data could be collected for a couple of weeks (for example, EMA data were collected for 4 weeks before treatment in Fisher et al., 2019). After data collection, the therapist and the client could independently draw a network of the symptoms and risk/protective factors. They could then discuss the data-driven and expectations-driven networks together to create a full picture of the psychopathological mechanisms of the client, and, based on this, personalize psychotherapy. Indeed, giving personalized feedback to patients has been suggested to increase motivation in mental health settings (Musiat et al., 2012), and may be a successful intervention to address, for example, problem drinking (Riper et al., 2009). The use of idiographic networks in clinical practice could be complemented with more simple statistics. Simple statistics are, for example, plots of the symptoms' averages and standard deviations over time. These can provide important information on the severity of the symptoms and how variables fluctuate over one day, one week, or longer periods of time.

To conclude, applications that allow to collect EMA data, estimate idiographic networks, integrate these networks with therapists' and clients' expectations and previous research, and visualize the networks, may be a promising new tool for clinical practice. These could be used to tailor communication in psychotherapy, inform treatment, provide personalized feedback, and improve client's motivation and adherence to therapy.

\section{Limitations and Strengths}


The current study had some limitations. First, items aggregation was relatively arbitrary. For example, we categorized feeling tired as a symptom of depression, but, according to DSM 5, this can also be a symptom of generalized anxiety disorder. Second, Dataset 1 did not meet the assumption of equal time-intervals between measurements. Both these limitations become problematic especially when interpreting the networks, however, this was not the aim of the current study, additionally, the estimated networks were in line with clinical expectations and previous research. Third, we simulated stationary normal data. Deviation from the assumptions of normality and stationarity may negatively influence GVAR performance. Fourth, we generated MCAR data. This means that our results are valid for planned missingness, but may not be valid for non-planned missingness, where MNAR data is expected.

This study also had two crucial strengths. We simulated data using real datasets measuring psychological variables, thus we could test the performance of GVAR to estimate psychological networks similar to those we would actually find in clinical settings. As estimation performance varies with varying network structures, running a simulation in the context of clinical psychology was of great importance. Additionally, we confirmed the stability of our findings by replicating them in two different datasets.

\section{Conclusions}

The aim of the present study was to provide recommendations for the application of personalized networks in clinical settings, with a focus on sample sizes and missing data handling. Sensitivity is low with small sample sizes that are feasible in clinical research and practice. It seems possible to retrieve the global structure of the network, but it is impossible to recover the full network. In particular, contemporaneous networks appears easier to retrieve compared to temporal networks. To estimate the temporal global structure with a number of time points between 75 and 100, it is advisable to include a small number of 
nodes, i.e., around 6 variables. Regarding our second question, both FIML and the Kalman filter are effective in addressing random item-level missing data, thus planned missingness and adaptive testing are valid methods to deal with missing data. Different strategies were identified to address the problem of low power, most of which are based on integrating the network estimated from the data with therapist's expertise, patient's expectations and previous research. A collaborative approach was proposed where idiographic networks based on data and previous knowledge are discussed with the patients to inform psychotherapy, tailor communication, provide personalized feedback, and improve client's motivation and adherence to treatment. 


\section{References}

Abegaz, F., \& Wit, E. (2013). Sparse time series chain graphical models for reconstructing genetic networks. Biostatistics, 14(3), 586-599. https://doi.org/10.1093/biostatistics/kxt005

Birnbaum, F., Lewis, D. M., Rosen, R., \& Ranney, M. L. (2015). Patient engagement and the design of digital health. Academic Emergency Medicine: Official Journal of the Society for Academic Emergency Medicine, 22(6), 754-756.

https://doi.org/10.1111/acem.12692

Borsboom, D. (2017). A network theory of mental disorders. World Psychiatry, 16(1), 5-13. https://doi.org/10.1002/wps.20375

Borsboom, D., \& Cramer, A. O. J. (2013). Network analysis: an integrative approach to the structure of psychopathology. Annual Review of Clinical Psychology, 9(1), 91-121. https://doi.org/10.1146/annurev-clinpsy-050212-185608

Burger, Epskamp, van der Veen, Dablander, Schoevers, Riese, \& Fried (in preparation). Models that matter for patients: Integrating clinical case formulation and idiographic network estimation.

Chen, J., \& Chen, Z. (2008). Extended Bayesian information criteria for model selection with large model spaces. Biometrika, 95(3), 759-771. https://doi.org/10.1093/biomet/asn034

Chevance, G., Perski, O., \& Hekler, E. B. (2020). Innovative methods for predicting and changing complex health behaviors: four propositions. PsyArXiv. https://doi.org/10.31234/osf.io/w6h78

Collins, L. M., Schafer, J. L., \& Kam, C.-M. (2001). A comparison of inclusive and restrictive strategies in modern missing data procedures. Psychological Methods, 6(4), 330-351. https://doi.org/10.1037/1082-989X.6.4.330 
Cramer, AngéLique O. J., \& Borsboom, D. (2015). Problems attract problems: A network perspective on mental disorders. Emerging Trends in the Social and Behavioral Sciences. https://doi.org/10.1002/9781118900772.etrds0264

Cramer, Angélique O. J., Waldorp, L. J., van der Maas, H. L. J., \& Borsboom, D. (2010). Comorbidity: a network perspective. The Behavioral and Brain Sciences, 33(2-3), 137-150. https://doi.org/10.1017/S0140525X09991567

de Ron, J., Fried, E. I., \& Epskamp, S. (2019). Psychological networks in clinical populations: a tutorial on the consequences of Berkson's bias. PsyArXiv. https://doi.org/10.31234/osf.io/5t8zw

DiFranza, J. R., Savageau, J. A., Fletcher, K., Ockene, J. K., Rigotti, N. A., McNeill, A. D., Coleman, M., \& Wood, C. (2004). Recollections and repercussions of the first inhaled cigarette. Addictive Behaviors, 29(2), 261-272. https://doi.org/10.1016/j.addbeh.2003.08.002

Eisen, S. V., Dickey, B., \& Sederer, L. I. (2000). A self-report symptom and problem rating scale to increase inpatients' involvement in treatment. Psychiatric Services, 51(3), 349-353. https://doi.org/10.1176/appi.ps.51.3.349

Enders, C., \& Bandalos, D. (2001). The relative performance of full information maximum likelihood estimation for missing data in structural equation models. Structural Equation Modeling: A Multidisciplinary Journal, 8(3), 430-457. https://doi.org/10.1207/S15328007SEM0803_5

Enders, C. K. (2001). A primer on maximum likelihood algorithms available for use with missing data. Structural Equation Modeling: A Multidisciplinary Journal, 8(1), 128141. https://doi.org/10.1207/S15328007SEM0801_7

Epskamp, S. (2019). ParSim: Parallel simulation studies (R package version 0.1.1) [Computer Software] Https://CRAN.R-project.org/package=parSim. 
Epskamp, S. (2020a). GraphicalVAR: Graphical VAR for experience sampling data. (R package version 0.2.3) [Computer Software] Https://CRAN.Rproject.org/package=graphicalVAR.

Epskamp, S. (2020b). Psychonetrics: Structural equation modeling and confirmatory network analysis. (R package version 0.7.6) [Computer Software] Http://psychonetrics.org/.

Epskamp, S. (2020c). Psychometric network models from time-series and panel data. Psychometrika, 85(1), 206-231. https://doi.org/10.1007/s11336-020-09697-3

Epskamp, S., Borsboom, D., \& Fried, E. I. (2018). Estimating psychological networks and their accuracy: a tutorial paper. Behavior Research Methods, 50(1), 195-212. https://doi.org/10.3758/s13428-017-0862-1

Epskamp, S., \& Fried, E. I. (2018). A tutorial on regularized partial correlation networks. Psychological Methods, 23(4), 617-634. https://doi.org/10.1037/met0000167

Epskamp, S., Kruis, J., \& Marsman, M. (2017). Estimating psychopathological networks: Be careful what you wish for. PLoS ONE, 12(6), Article e0179891. https://doi.org/10.1371/journal.pone.0179891

Epskamp, S., Rhemtulla, M., \& Borsboom, D. (2017). Generalized network psychometrics: Combining network and latent variable models. Psychometrika, 82(4), 904-927. https://doi.org/10.1007/s11336-017-9557-x

Epskamp, S., van Borkulo, C. D., van der Veen, D. C., Servaas, M. N., Isvoranu, A.-M., Riese, H., \& Cramer, A. O. J. (2018). Personalized network modeling in psychopathology: the importance of contemporaneous and temporal connections. Clinical Psychological Science, 6(3), 416-427. https://doi.org/10.1177/2167702617744325 
Epskamp, S., Waldorp, L. J., Mõttus, R., \& Borsboom, D. (2018). The gaussian graphical model in cross-sectional and time-series data. Multivariate Behavioral Research, 53(4), 453-480. https://doi.org/10.1080/00273171.2018.1454823

Epskamp, S., Deserno, M. K., \& Bringmann, L. F. (2019). MlVAR: Multi-level vector autoregression (R package version 0.4.4) [Computer Software] Https://CRAN.Rproject.org/package $=m l V A R$.

Fisher, A. J., \& Bosley, H. G. (2015). Personalized assessment and treatment of depression. Current Opinion in Psychology, 4, 67-74. https://doi.org/10.1016/j.copsyc.2015.03.031

Fisher, A. J., Bosley, H. G., Fernandez, K. C., Reeves, J. W., Soyster, P. D., Diamond, A. E., \& Barkin, J. (2019). Open trial of a personalized modular treatment for mood and anxiety. Behaviour Research and Therapy, 116, 69-79. https://doi.org/10.1016/j.brat.2019.01.010

Fisher, A. J., \& Boswell, J. F. (2016). Enhancing the personalization of psychotherapy with dynamic assessment and modeling. Assessment, 23(4), 496-506. https://doi.org/10.1177/1073191116638735

Fisher, A. J., Medaglia, J. D., \& Jeronimus, B. F. (2018). Lack of group-to-individual generalizability is a threat to human subjects research. Proceedings of the National Academy of Sciences, 115(27), E6106-E6115. https://doi.org/10.1073/pnas.1711978115

Foygel, R., \& Drton, M. (2010). Extended bayesian information criteria for gaussian graphical models. In J. D. Lafferty, C. K. I. Williams, J. Shawe-Taylor, R. S. Zemel, \& A. Culotta (Eds.), Advances in Neural Information Processing Systems 23 (pp. 604-612). Curran Associates, Inc. http://papers.nips.cc/paper/4087-extendedbayesian-information-criteria-for-gaussian-graphical-models.pdf 
Fried, E. I., Papanikolaou, F., Epskamp, S. (2020). Mental health and social contact during the COVID-19 pandemic: an ecological momentary assessment study. PsyArXiv. https://doi.org/10.31234/osf.io/36xkp

Fried, E. I., van Borkulo, C. D., Cramer, A. O. J., Boschloo, L., Schoevers, R. A., \& Borsboom, D. (2017). Mental disorders as networks of problems: a review of recent insights. Social Psychiatry and Psychiatric Epidemiology, 52(1), 1-10. https://doi.org/10.1007/s00127-016-1319-z

Frumkin, M. R., Piccirillo, M. L., Beck, E. D., Grossman, J. T., \& Rodebaugh, T. L. (2020). Feasibility and utility of idiographic models in the clinic: a pilot study. Psychotherapy Research, 1-15. https://doi.org/10.1080/10503307.2020.1805133

Graham, J. W. (2009). Missing data analysis: Making it work in the real world. Annual Review of Psychology, 60(1), 549-576. https://doi.org/10.1146/annurev.psych.58.110405.085530

Graham, J. W., Taylor, B. J., \& Cumsille, P. E. (2001). Planned missing-data designs in analysis of change. In L. M. Collins \& A. G. Sayer (Eds.), Decade of behavior. New methods for the analysis of change (p. 335-353). American Psychological Association. https://doi.org/10.1037/10409-011

Graham, J. W., Taylor, B. J., Olchowski, A. E., \& Cumsille, P. E. (2006). Planned missing data designs in psychological research. Psychological Methods, 11(4), 323-343. https://doi.org/10.1037/1082-989X.11.4.323

Hamaker, E. L., \& Grasman, R. P. P. P. (2012). Regime switching state-space models applied to psychological processes: Handling missing data and making inferences. Psychometrika, 77(2), 400-422. https://doi.org/10.1007/s11336-012-9254-8 
Hamaker, Ellen L., \& Wichers, M. (2017). No time like the present: Discovering the hidden dynamics in intensive longitudinal data. Current Directions in Psychological Science, 26(1), 10-15. https://doi.org/10.1177/0963721416666518

Harel, O., Stratton, J., \& Aseltine, R. (2015). Designed missingness to better estimate efficacy of behavioral studies-application to suicide prevention trials. Journal of Medical Statistics and Informatics, 3(1), Article 2. https://doi.org/10.7243/2053-7662$3-2$

Harvey, A. C. (1989). Forecasting, structural time series models and the Kalman filter. Cambridge University Press.

Hawkins, R. P., Kreuter, M., Resnicow, K., Fishbein, M., \& Dijkstra, A. (2008). Understanding tailoring in communicating about health. Health Education Research, 23(3), 454-466. https://doi.org/10.1093/her/cyn004

Hofmann, S. G., Curtiss, J. E., \& Hayes, S. C. (2020). Beyond linear mediation: Toward a dynamic network approach to study treatment processes. Clinical Psychology Review, 76, Article 101824. https://doi.org/10.1016/j.cpr.2020.101824

Hofmann, S. G., Curtiss, J., \& McNally, R. J. (2016). A complex network perspective on clinical science. Perspectives on Psychological Science, 11(5), 597-605. https://doi.org/10.1177/1745691616639283

Howard, K. I., Moras, K., Brill, P. L., Martinovich, Z., \& Lutz, W. (1996). Evaluation of psychotherapy: efficacy, effectiveness, and patient progress. American Psychologist, 51(10), 1059-1064. https://doi.org/10.1037/0003-066X.51.10.1059

Iorfino, F., Cross, S. P., Davenport, T., Carpenter, J. S., Scott, E., Shiran, S., \& Hickie, I. B. (2019). A digital platform designed for youth mental health services to deliver personalized and measurement-based care. Frontiers in Psychiatry, 10. https://doi.org/10.3389/fpsyt.2019.00595 
Jones, A., Remmerswaal, D., Verveer, I., Robinson, E., Franken, I. H. A., Wen, C. K. F., \& Field, M. (2019). Compliance with ecological momentary assessment protocols in substance users: a meta-analysis. Addiction, 114(4), 609-619. https://doi.org/10.1111/add.14503

Kroeze, R., van der Veen, D. C., Servaas, M. N., Bastiaansen, J. A., Voshaar, R. C. O. V., Borsboom, D., Ruhe, H. G., Schoevers, R. A., \& Riese, H. (2017). Personalized feedback on symptom dynamics of psychopathology: a proof-of-principle study. Journal for Person-Oriented Research, 3(1), 1-11. https://doi.org/10.17505/jpor.2017.01

Larsen, R. (2011). Missing data imputation versus full information maximum likelihood with second-level dependencies. Structural Equation Modeling: A Multidisciplinary Journal, 18(4), 649-662. https://doi.org/10.1080/10705511.2011.607721

McLean, D. C., Nakamura, J., \& Csikszentmihalyi, M. (2017). Explaining system missing: missing data and experience sampling method. Social Psychological and Personality Science, 8(4), 434-441. https://doi.org/10.1177/1948550617708015

McNally, R. J. (2016). Can network analysis transform psychopathology? Behaviour Research and Therapy, 86, 95-104. https://doi.org/10.1016/j.brat.2016.06.006

Mohr, D. C., Zhang, M., \& Schueller, S. M. (2017). Personal sensing: Understanding mental health using ubiquitous sensors and machine learning. Annual Review of Clinical Psychology, 13, 23-47. https://doi.org/10.1146/annurev-clinpsy-032816-044949

Molenaar, P. C. M. (2004). A manifesto on psychology as idiographic science: Bringing the person back into scientific psychology, this time forever. Measurement: Interdisciplinary Research \& Perspective, 2(4), 201-218. https://doi.org/10.1207/s15366359mea0204_1 
Moritz, S., \& Bartz-Beielstein, T. (2017). ImputeTS: Time series missing value imputation in R. The R Journal, 9(1), 207-218. https://doi.org/10.32614/RJ-2017-009

Musiat, P., Hoffmann, L., \& Schmidt, U. (2012). Personalised computerised feedback in Emental health. Journal of Mental Health, 21(4), 346-354. https://doi.org/10.3109/09638237.2011.648347

Neath, A. A., \& Cavanaugh, J. E. (2012). The Bayesian information criterion: background, derivation, and applications. Wiley Interdisciplinary Reviews: Computational Statistics, 4(2), 199-203. https://onlinelibrary.wiley.com/doi/full/10.1002/wics.199

Ono, M., Schneider, S., Junghaenel, D. U., \& Stone, A. A. (2019). What affects the completion of ecological momentary assessments in chronic pain research? An individual patient data meta-analysis. Journal of Medical Internet Research, 21(2), Article e11398. https://doi.org/10.2196/11398

Patrick, K., Hekler, E. B., Estrin, D., Mohr, D. C., Riper, H., Crane, D., Godino, J., \& Riley, W. T. (2016). The pace of technologic change: implications for digital health behavior intervention research. American Journal of Preventive Medicine, 51(5), 816-824. https://doi.org/10.1016/j.amepre.2016.05.001

Piasecki, T. M., Jahng, S., Wood, P. K., Robertson, B. M., Epler, A. J., Cronk, N. J., Rohrbaugh, J. W., Heath, A. C., Shiffman, S., \& Sher, K. J. (2011). The subjective effects of alcohol-tobacco co-use: an ecological momentary assessment investigation. Journal of Abnormal Psychology, 120(3), 557-571. https://doi.org/10.1037/a0023033

Piccirillo, M. L., \& Rodebaugh, T. L. (2019). Foundations of idiographic methods in psychology and applications for psychotherapy. Clinical Psychology Review, 71, 90100. https://doi.org/10.1016/j.cpr.2019.01.002

Pomerleau, C. S., Pomerleau, O. F., Namenek, R. J., \& Marks, J. L. (1999). Initial exposure to nicotine in college-age women smokers and never-smokers: a replication and 
extension. Journal of Addictive Diseases, 18(3), 13-19.

https://doi.org/10.1300/J069v18n03_02

Pomerleau, O. F., Pomerleau, C. S., \& Namenek, R. J. (1998). Early experiences with tobacco among women smokers, ex-smokers, and never-smokers. Addiction, 93(4), 595-599. https://doi.org/10.1046/j.1360-0443.1998.93459515.x

Rintala, A., Wampers, M., Myin-Germeys, I., \& Viechtbauer, W. (2019). Response compliance and predictors thereof in studies using the experience sampling method. Psychological Assessment, 31(2), 226-235. https://doi.org/10.1037/pas0000662

Riper, H., van Straten, A., Keuken, M., Smit, F., Schippers, G., \& Cuijpers, P. (2009). Curbing problem drinking with personalized-feedback interventions: a meta-analysis. American Journal of Preventive Medicine, 36(3), 247-255. https://doi.org/10.1016/j.amepre.2008.10.016

Robinaugh, D. J., Brown, M. L., Losiewicz, O. M., Jones, P. J., Marques, L., \& Baker, A. W. (2020). Towards a precision psychiatry approach to anxiety disorders with ecological momentary assessment: the example of panic disorder. General Psychiatry, 33(1), Article e100161. https://doi.org/10.1136/gpsych-2019-100161

Robinaugh, D. J., Hoekstra, R. H. A., Toner, E. R., \& Borsboom, D. (2020). The network approach to psychopathology: A review of the literature 2008-2018 and an agenda for future research. Psychological Medicine, 50(3), 353-366.

https://doi.org/10.1017/S0033291719003404

Rothman, A. J., Levina, E., \& Zhu, J. (2010). Sparse multivariate regression with covariance estimation. Journal of Computational and Graphical Statistics, 19(4), 947-962. https://doi.org/10.1198/jcgs.2010.09188

Schafer, J. L., \& Graham, J. W. (2002). Missing data: our view of the state of the art. Psychological Methods, 7(2), 147-177. https://doi.org/10.1037/1082-989X.7.2.147 
Scott, K., \& Lewis, C. C. (2015). Using measurement-based care to enhance any treatment. Cognitive and Behavioral Practice, 22(1), 49-59. https://doi.org/10.1016/j.cbpra.2014.01.010

Serre, F., Fatseas, M., Swendsen, J., \& Auriacombe, M. (2015). Ecological momentary assessment in the investigation of craving and substance use in daily life: a systematic review. Drug and Alcohol Dependence, 148, 1-20. https://doi.org/10.1016/j.drugalcdep.2014.12.024

Shiffman, S., Stone, A. A., \& Hufford, M. R. (2008). Ecological Momentary Assessment. Annual Review of Clinical Psychology, 4(1), 1-32. https://doi.org/10.1146/annurev.clinpsy.3.022806.091415

Shin, T., Davison, M. L., \& Long, J. D. (2017). Maximum likelihood versus multiple imputation for missing data in small longitudinal samples with nonnormality. Psychological Methods, 22(3), 426-449. https://doi.org/10.1037/met0000094

Spencer, J., Goode, J., Penix, E. A., Trusty, W., \& Swift, J. K. (2019). Developing a collaborative relationship with clients during the initial sessions of psychotherapy. Psychotherapy, 56(1), 7-10. https://doi.org/10.1037/pst0000208

Trull, T. J., \& Ebner-Priemer, U. (2013). Ambulatory assessment. Annual Review of Clinical Psychology, 9(1), 151-176. https://doi.org/10.1146/annurev-clinpsy-050212-185510 Tryon, G. S., Birch, S. E., \& Verkuilen, J. (2018). Meta-analyses of the relation of goal consensus and collaboration to psychotherapy outcome. Psychotherapy, 55(4), 372383. https://doi.org/10.1037/pst0000170

von Klipstein, L., Riese, H., Servaas, M. N., \& Schoevers, R. A. (2020). Using personspecific networks in psychotherapy: challenges, limitations, and how we could use them anyway. BMC medicine, 18(1), 1-8.

Wen, C. K. F., Schneider, S., Stone, A. A., \& Spruijt-Metz, D. (2017). Compliance with 
mobile ecological momentary assessment protocols in children and adolescents: a systematic review and meta-analysis. Journal of Medical Internet Research, 19(4), Article e132. https://doi.org/10.2196/jmir.6641

Wichers, M., Groot, P. C., Psychosystems, ESM, \& EWS Group. (2016). Critical slowing down as a personalized early warning signal for depression. Psychotherapy and Psychosomatics, 85(2), 114-116. https://doi.org/10.1159/000441458

Wild, B., Eichler, M., Friederich, H.-C., Hartmann, M., Zipfel, S., \& Herzog, W. (2010). A graphical vector autoregressive modelling approach to the analysis of electronic diary data. BMC Medical Research Methodology, 10(1), Article 28. https://doi.org/10.1186/1471-2288-10-28

Wilhelm, P., \& Schoebi, D. (2007). Assessing mood in daily life: structural validity, sensitivity to change, and reliability of a short-scale to measure three basic dimensions of mood. European Journal of Psychological Assessment, 23(4), 258267. https://doi.org/10.1027/1015-5759.23.4.258

Wright, A. G. C., \& Woods, W. C. (2020). Personalized models of psychopathology. Annual Review of Clinical Psychology, 16(1), 49-74. https://doi.org/10.1146/annurevclinpsy-102419-125032

Yuan, K.-H., Yang-Wallentin, F., \& Bentler, P. M. (2012). ML versus MI for missing data with violation of distribution conditions. Sociological Methods \& Research, 41(4), 598-629. https://doi.org/10.1177/0049124112460373 